\title{
3D MODELING OF ION RIBBON BEAM FOCUSING AND ACCELERATION IN UNDULATOR LINAC
}

\author{
E.S. Masunov $^{*}$, S.M. Polozov, A.S. Roshal, MEPHI, RUSSIA
}

\section{Abstract}

One of the possible versions of undulator linear accelerator (UNDULAC-E) using a ribbon beam is discussed. The computer 3D simulation of the beam dynamics is fulfilled. The possibility of simultaneous acceleration of both positive and negative ions in undulator linac is shown. The influence of the fundamental and higher space harmonics of the RF field on ion ribbon beam dynamics in UNDULAC-E is analyzed. The optimal parameters of the accelerator are calculated in order to reach the higher transmission. Simulation results are presented.

\section{INTRODUCTION}

Earlier the idea to apply a combination of a plane electrostatic undulator and radio frequency fields for acceleration and focusing of intense ion beams with low injection energy was suggested and theoretically investigated in [1]. The current in such undulator linac (UNDULAC-E) can be increased by using a ribbon beam [2]. One of the possible versions of UNDULAC-E with the ribbon beam was discussed in [3]. It was shown that the focusing force is normal to the plane surface of the ribbon beam. The beam focusing in another transverse direction can be created by a nonuniform distribution of the undulator field along the ribbon width (Fig. 1). In [4] there was suggested to change the form of electrodes for two-dimensional transverse beam focusing. Using the smooth approximation method it was found the electrodes shape and the amplitudes of the undulator and RF fields for beam focusing.

The other factor limiting the beam intensity in the undulator linac is a space charge force. The influence of the space charge effects on the ribbon beam focusing was investigated in [4]. The computer simulation of ion dynamics in the undulator, RF and self-consistent Coulomb fields was done by some approximations. The most important of it was the assumption that all ions had the same axial velocity as the synchronous particle and its spread was neglected. The initial transverse velocities was supposed to be normally distributed with relatively small standard deviation. This crude method is good for bunched beam when the coupling resonance is absent. It was shown that the electrostatic shielding of the space charge field decreases Coulomb defocusing of the particles in the narrow accelerating channel. The results of the simulation for intense ion beam dynamics demonstrated the possibility to accelerate the high current beam in UNDULAC-E.

The other important way for increasing of the beam intensity is using the space charge compensation $[1,5]$. In the UNDULAC the accelerating force does not depend on a sign of the particle charge and the averaged motion of the positive and the negative charges are identical. The $e Z$ and $e Z^{+}$ions will be inside the same separatrix (bunch) and the current limit of the ion beam can be substantially increased. Space charge compensation effect can be disturbed if the transverse emittance has been changing asymmetrical for $e Z^{+}$and $e Z$ and halo phenomenon has been forming. In this paper the behavior of transverse and longitudinal dynamics are investigated in nonlinear RF and undulator fields.

\section{SMOOTH APPROXIMATION FOR MOTION EQUATION}

At first let us consider the ions $3 \mathrm{D}$ dynamics in the RF and the undulator fields using the smooth approximation. In the periodic structure the RF potential $U_{v}$ and the electrostatic periodic potential $U_{o}$ are delivered to the same electrodes (Fig.1.). The both potentials can be represented as the sum of the space harmonics:

$$
\begin{aligned}
& U_{v}=-\left(\Phi_{v}^{(0)}(y)+\sum_{n=1}^{\infty} \Phi_{v}^{(2 n)}(x, y) \cos \left(4 \pi n \int d z / D\right)\right) \sin \omega t, \\
& U_{o}=-\sum_{n=1}^{\infty} \Phi_{o}^{(n)}(x, y) \cos \left(2 \pi n \int d z / D\right),
\end{aligned}
$$

where $\Phi_{o, v}^{(n)}(x, y)=\Phi_{o, v}^{(n)} \cosh \left(n k_{x} x\right) \sinh \left(n k_{y} y\right)$, $\mathrm{D}$ is the undulator field $\mathrm{z}$-depended period.

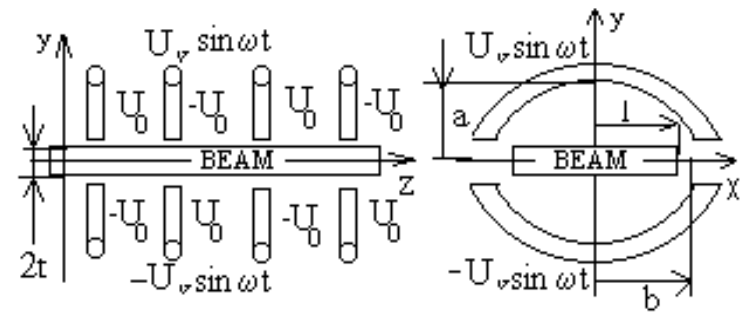

Figure 1: Cross section of the accelerator system.

The fundamental and the higher space harmonics of the $\mathrm{RF}$ and the undulator fields are nonsynchronous with the beam. The mechanism of the acceleration and the

*E-mail: masunov@dinus.mephi.ru 
focusing is produced by a combined wave field, resulted from a superposition of the first space harmonic of the undulator field and the zero space harmonic of the transverse RF field. The interaction of the accelerated particle with the nonsynchronous harmonics fields can be analyzed by averaging method. In accordance with that, the trajectory of a ion may be represented as a combination of rapidly oscillations $\boldsymbol{r}(\boldsymbol{t})$ and slow variation $\boldsymbol{R}(\boldsymbol{t})$. After the procedure of averaging over rapidly oscillations one can derive the expression for effective potential function $U_{\text {eff }}$ describing the averaged particle motion [1,4]. Using this effective potential $U_{\text {eff }}$ the equation for the synchronous particle velocity $\beta_{s}=v_{s} / c$ can be written as

$$
\frac{d \beta_{s}^{3}}{d z}=\frac{3 e^{2} Z^{2}}{4 \pi} \frac{E_{o}^{(1)} \cdot E_{v}^{(0)}}{m^{2} c^{4}} \cdot \cos \varphi_{s}
$$

where $E_{o}^{(l)}$ and $E_{v}{ }^{(0)}$ are the amplitudes of the first undulator field harmonic and the zero RF field harmonic and $\varphi=\frac{2 \pi}{\lambda} \int \frac{d z}{\beta_{s}}-\omega t+\delta$ is the particle phase in the combined wave.

The efficient capture and bunching of the beam can be obtained by the adiabatic growth of the amplitudes $E_{o}$ and $E_{v}$ along the longitudinal axis and the corresponding increase of undulator period $D=\beta / \lambda$ to maintain the beam synchronism with the combined wave field. The choice of the function $E_{o}$ and $E_{v}$ is not arbitrary because simultaneously with acceleration it is necessary to keep up the transverse focusing of the beam. The analysis of the transverse beam dynamics by means of $U_{\text {eff }}$ shows that the focusing along $y$ direction is possible if $2 E_{o}>E_{v} \sin \varphi$ and the focusing along $x$ direction is possible if $E_{o}>E_{v} \sin \varphi$ The accurate analysis of beam 3D dynamics in UNDULAC can be made only by numerical simulation.

\section{BEAM DYNAMICS IN UNDULAC}

We are discussed UNDULAC design for deuterium ions $D^{-}$and $D^{+}$. The UNDULAC consists of the gentle buncher (GB) and acceleration area. The transverse sizes of the channel $2 a=0.8 \mathrm{~cm}, 2 b=20 \mathrm{~cm}$. Computer simulation of two component ion beam, consisting of $D^{-}$ and $D^{+}$was carried out by a macroparticle method. The input characteristics of $D^{-}$and $D^{+}$are identical. The ribbon beam had the input thickness $2 t=0.5 \mathrm{~cm}$ and the input width $2 l=11.5 \mathrm{~cm}$. The input transverse velocity has deviation $\sigma=0.01$.The input/output energy are respectively $150 \mathrm{keV} / 1 \mathrm{MeV}$ and the operating frequency is set at 200 MHz. The amplitude of RF field $E_{v}<240 \mathrm{kV} / \mathrm{cm}$. In the buncher the fundamental harmonic amplitudes of RF and undulator fields were gradually grown as $\sin \left[\pi\left(z+z_{0}\right) / 2\left(z_{0}+L_{f}\right)\right]$ when $0 \leq z \leq L_{f}$. The synchronous phase $\varphi_{\mathrm{s}}$ was linearly decreased from $\pi / 2$ to $\pi / 4$ when $0 \leq$ $z \leq L_{\varphi}$. In the acceleration area $E_{o, v}$ and $\varphi_{\mathrm{s}}$ are constant. The dynamics has been calculated by using these parameters to reach the higher transmission. For the basic variant $E_{v}=E_{o}=150 \mathrm{kV} / \mathrm{cm}$, the length of the accelerator $L=2.5 \mathrm{~m}$.

The numerical simulation shows that the beam capture and the transmission depend on the choice of $L_{\varphi}$ and $L_{\mathrm{f}}$. The transmission coefficient $K$ increases from 0.74 to 0.9 when $L_{\varphi}$ and $L_{f}$ are enhanced from 40 to $100 \mathrm{~cm}$ but the acceleration gradient decreases. Some results for $K$ and the output energy $W$ are listed in Table 1 .

Table 1.

\begin{tabular}{|c|c|c|c|c|c|}
\hline \multicolumn{2}{|c|}{$L_{f} \mathrm{~cm}$} & 40 & 50 & 75 & 100 \\
\hline \multirow{2}{*}{40} & $K, \%$ & 73.8 & 75.5 & 76.7 & 77.6 \\
\cline { 2 - 6 } & $W, \mathrm{keV}$ & 1070 & 1058 & 1030 & 991 \\
\hline \multirow{2}{*}{50} & $K, \%$ & 75.7 & 78.0 & 78.1 & 80.0 \\
\cline { 2 - 6 } & $W, \mathrm{keV}$ & 1056 & 1050 & 1020 & 985 \\
\hline \multirow{2}{*}{75} & $K, \%$ & 73.8 & 78.9 & 81.4 & 84.0 \\
\cline { 2 - 6 } & $W, \mathrm{keV}$ & 1027 & 1018 & 1005 & 971 \\
\hline \multirow{2}{*}{100} & $K, \%$ & 71.5 & 76.3 & 80.3 & 89.9 \\
\cline { 2 - 6 } & $W, \mathrm{keV}$ & 991 & 988 & 973 & 949 \\
\hline
\end{tabular}

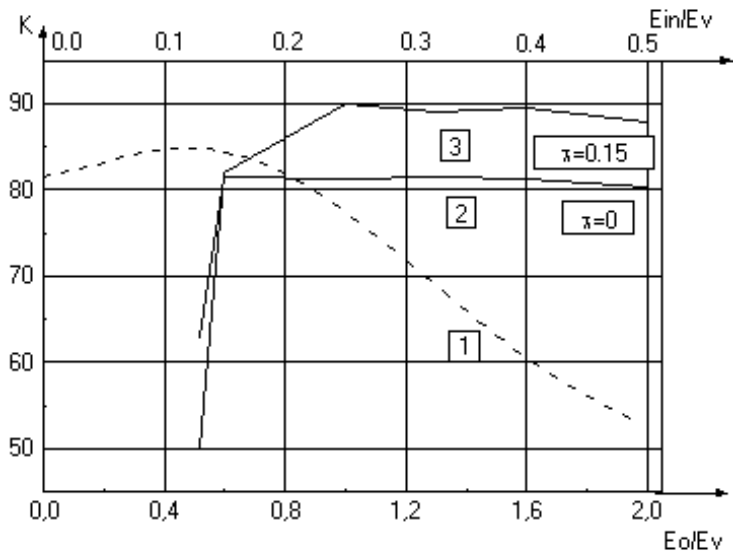

Figure 2: Transmission coefficient $K$ versus field parameters.

The modeling shows that the input transverse RF field amplitude $E_{\text {in }}$ must be small (Fig. 2, curve 1). The transmission coefficient $K>0.8$ if $0<E_{i n} / E_{v}<0.22$ and $E_{o}=E_{v}$. Then $K$ rapidly decreases if $E_{i n} / E_{\max }>0.3$. The most of the ions losses are located at the beginning of the GB section. These losses are due to the longitudinal dynamics The transverse dynamics and the focusing effect depend on the undulator field amplitude. For small $E_{o}$ focusing is absent and $K$ is very small (Fig. 2, curve 2). The beam transmission rapidly increases when $0.46<E_{d} / E_{v}<0.5$. If $E_{d} / E_{v}>0.5$ and the value of $E_{o} \cdot E_{v}$ is given constant the coefficient $K$ has the limit 0.81 for $L_{f}=0.75 \mathrm{~m}$.

The next figure gives simulation results for two cases:

a) $E_{o}=150 \mathrm{kV} / \mathrm{cm}, E_{v}=150 \mathrm{kV} / \mathrm{cm}, E_{o} / E_{v}=1.0$;

b) $E_{o}=125 \mathrm{kV} / \mathrm{cm}, E_{v}=180 \mathrm{kV} / \mathrm{cm}, E_{d} / E_{v}=0.7$. 
All output characteristics of $D^{+}$and $D^{-}$are identical and the transverse emittances have been changing symmetric. This fact illustrates that the space charge compensation effect is preserved.
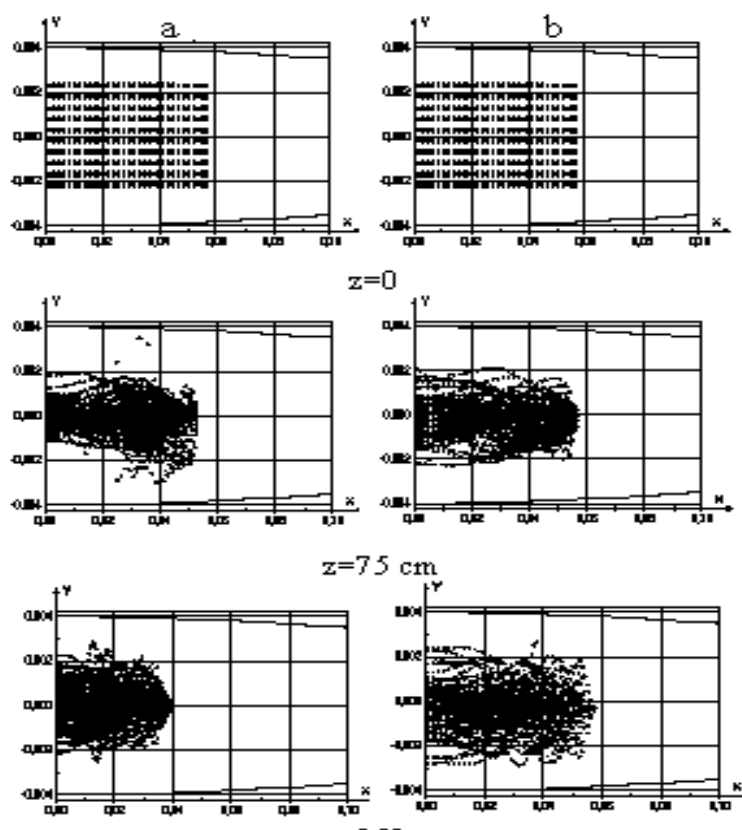

$\mathrm{z}=75 \mathrm{~cm}$
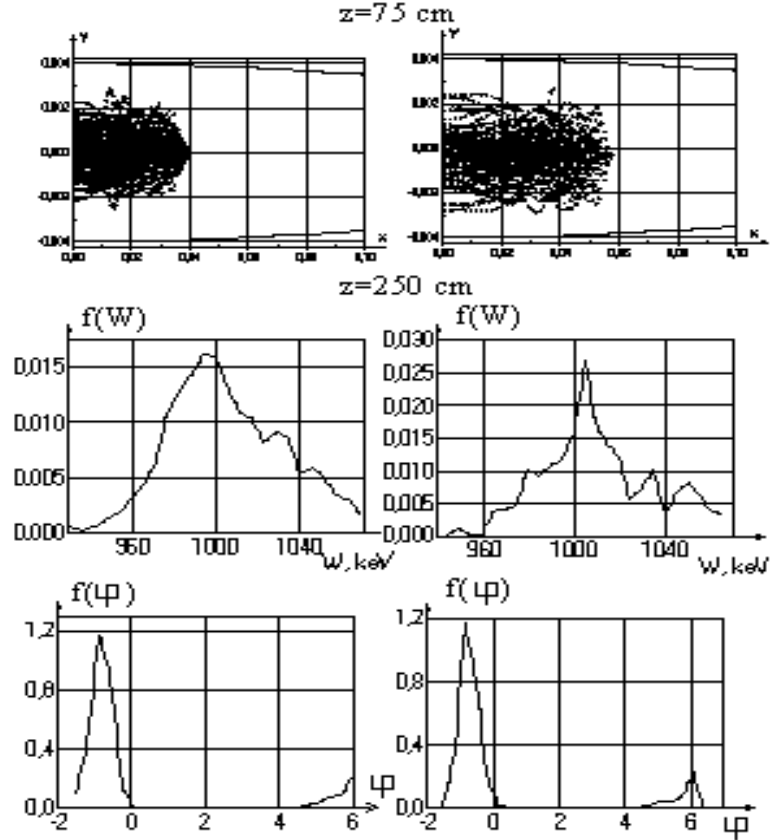

$f(\varphi)$

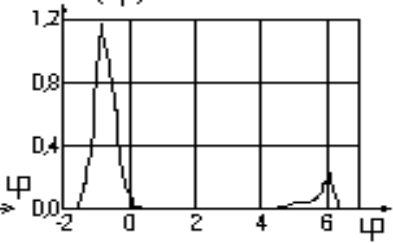

Figure 3: Beam cross-section at different $z$; density functions of output energy $f(W)$ and output phase $f(\varphi)$.

\section{INFLUENCE OF SECOND SPACE HARMONIC OF RF FIELD}

The higher space harmonics of RF and undulator fields may influence on ion ribbon beam dynamics in UNDULAC. Here we restrict ourselves by the consideration of the second RF harmonic. Using the smooth approximation the new effective potential $U_{\text {eff }}$ can be obtained. The shape of this effective potential deforms significantly. The analysis of the $U_{\text {eff }}$ shows that the phase capture and the acceleration gradient are increasing in this case. The transmission coefficient $K$ has maximum when $\chi=E_{v}^{(2)} / E_{v}^{(0)}=0.15$ (Fig. 4). The dependence of $K$ on the $E_{d} / E_{v}$ for $\chi=0.15$ is been imaged in Fig. 2, curve 3. The limit of $K=0.9$ if $E_{0} \geq E_{v}$ and $L_{f}=0.75 \mathrm{~m}$. The transverse emittances for $D^{+}$and $D^{-}$are identical too and the space charge compensation effect is preserved.

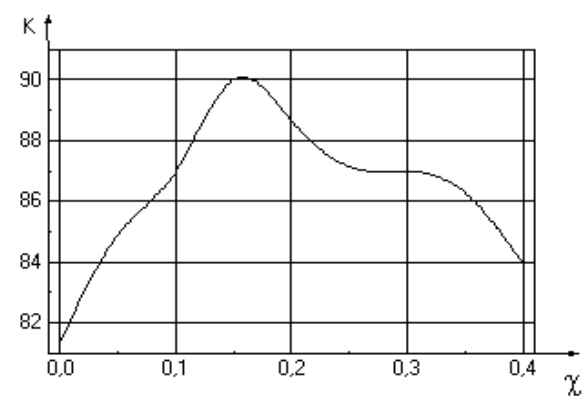

Figure 4: Transmission coefficient $K$ versus $\chi$.

\section{CONCLUSION}

The computer 3D simulation of the ribbon beam dynamics in the undulator linac is fulfilled. The optimal parameters of the UNDULAC are calculated. A preliminary design leading to the ion beam transmission larger than $90 \%$ is presented. The possibility of simultaneous acceleration of $D^{+}$and $D^{-}$ions in the UNDULAC is shown. It is proved that space overlapping of the positively and negatively charged ion beams allows to obtain space charge compensation. Simulation results for the design of the undulator linac with two component ion beam are presented.

\section{REFERENCES}

[1] E.S. Masunov, Zh. Tekh. Fiz. vol.60, p 152 (1990)

[2] E.S. Masunov, Proc. of the XVIII International Linac Conf., Geneva, Switzerland, CERN 96-07, 1996, vol.2, p.487-489

[3] E.S.Masunov, A.P.Novikov, Proc. 1991 Part. Accel. Conf., San Francisco, vol.5, p.3177 (1991)

[4] E.S. Masunov, A.S. Roshal, Proc. 1997 Part. Accel. Conf., Vancouver, p.2835 (1997)

[5] E.S. Masunov, A.P. Novicov, Proc. IV Europe Part. Accel. Conf., London, v. 2, p. 1171 (1994) 